\title{
REFINED VERSION OF HASSE'S SATZ 45 ON CLASS NUMBER PARITY
}

\author{
By \\ Humio ICHIMURA
}

\begin{abstract}
For an imaginary abelian field $K$, Hasse [3, Satz 45] obtained a criterion for the relative class number to be odd in terms of the narrow class number of the maximal real subfield $K^{+}$and the prime numbers which ramify in $K$, by using the analytic class number formula. In [4], we gave a refined version $(=" \Delta \Delta$-decomposed version") of Satz 45 by an algebraic method. In this paper, we give one more algebraic proof of the refined version.
\end{abstract}

\section{Introduction}

For a number field $N$, let $h_{N}$ denote the class number of $N$. When $N$ is an imaginary abelian field with the maximal real subfield $N^{+}$, we write $h_{N}^{+}=h_{N^{+}}$ and put $h_{N}^{-}=h_{N} / h_{N}^{+}$. Further, let $\tilde{h}_{N}^{+}$be the class number of $N^{+}$in the narrow sense. Let $k / \mathbf{Q}$ be an imaginary abelian extension of 2-power degree, and $F / \mathbf{Q}$ a real abelian extension with $2 \nmid[F: \mathbf{Q}]$, and put $K=F k$. In [3, Satz 45], Hasse proved the following theorem.

THEOREM 1. Under the above setting, assume further that the extension $K / \mathbf{Q}$ is cyclic. Then $h_{K}^{-}$is odd if and only if (i) $\tilde{h}_{K}^{+}$is odd, (ii) exactly one prime number ramifies in $k / \mathbf{Q}$, say $p$, and (iii) the prime number $p$ does not split in $F / \mathbf{Q}$.

When $F=\mathbf{Q}$, we immediately obtain the following corollary from Satz 45 and Washington [11, Theorem 10.4(b)].

Corollary 1. For an imaginary cyclic extension $k / \mathbf{Q}$ of 2-power degree, $h_{k}^{-}$ is odd if and only if exactly one prime number ramifies in $k$.

2010 Mathematics Subject Classification: Primary 11R18; Secondary 11R29.

Key words and phrases: Class number parity, abelian field, reflection argument. Received January 14, 2014. 
In what follows, we do not assume that $K / \mathbf{Q}$ is cyclic. Using class field theory, we can easily show that the ratios $h_{K}^{-} / h_{k}^{-}$and $\tilde{h}_{K}^{+} / \tilde{h}_{k}^{+}$are integers. In view of the above results, one is naturally interested in the parity of $h_{K}^{-} / h_{k}^{-}$, which is the subject of this note.

Hasse proved Theorem 1 heavily using the analytic class number formula. Recently, Conner and Hurrelbrink [2, Theorem 13.8] gave a purely algebraic proof of Theorem 1 using (i) their exact hexagon involving the cohomology groups $H^{i}\left(K / K^{+}, M\right)$ with $i=0,1$ where $M$ is the group of units or the ideal class group of $K$ and (ii) some fundamental properties of local norm residue symbols. In [4, Corollary 2], sharpening the method in [2], we obtained the following refined version of Theorem 1.

Let $\Delta=\operatorname{Gal}(F / \mathbf{Q})=\operatorname{Gal}(K / k)$. For a number field $N$, we denote by $A_{N}$ and $A_{N, \infty}$ the 2-parts of the ideal class group and the narrow class group of $N$, respectively. We put $A=A_{K}, A^{+}=A_{K^{+}}$and $A_{\infty}^{+}=A_{K^{+}, \infty}$ for brevity. We define the minus class group $A^{-}=A_{K}^{-}$to be the kernel of the norm map $A \rightarrow A^{+}$. We regard the above groups as modules over the group ring $\mathbf{Z}_{2}[\Delta]$. Let $\varphi$ be a nontrivial $\overline{\mathbf{Q}}_{2}$-valued character of $\Delta$, which we often regard as a primitive Dirichlet character. Here, $\mathbf{Z}_{2}$ denotes the ring of 2-adic integers and $\overline{\mathbf{Q}}_{2}$ a fixed algebraic closure of the 2-adic rationals $\mathbf{Q}_{2}$. For a $\mathbf{Z}_{2}[\Delta]$-module $X, X(\varphi)$ denotes the $\varphi$-component of $X$. (See $\S 2$, for the definition of the $\varphi$-component.) Let $S$ be the set of prime numbers $p$ such that a prime divisor of $k^{+}$over $p$ ramifies in $k$.

THEOREM 2. Under the above setting, we have $A^{-}(\varphi)=\{0\}$ if and only if (i) $A_{\infty}^{+}(\varphi)=\{0\}$ and (ii) $\varphi(p) \neq 1$ for any prime number $p \in S$.

COROLlaRY 2. The ratio $h_{K}^{-} / h_{k}^{-}$is odd if and only if (i) the ratio $\tilde{h}_{K}^{+} / \tilde{h}_{k}^{+}$is odd and (ii) no prime number $p$ in $S$ splits in $F$.

The main purpose of this paper is to give one more algebraic proof of Theorem 2 using a classical reflection argument. Further, we apply Theorem 2 to show that the 2-part of the class group of the cyclotomic $\mathbf{Z}_{2}$-extension over a certain imaginary abelian field is trivial (Theorem 3). We show Theorem 2 in $\S 4$ after some preliminaries in $\S 2$ and 3 . In $\S 5$, we show Theorem 3 .

REMARK. In some cases, there are two different proofs for an assertion on the 2-part of the ideal class group. For instance, a theorem of Armitage 
and Fröhlich [1] was generalized by Taylor [10] and Oriat [9] in two different ways. Taylor used some properties of norm residue symbols, while Oriat used a reflection argument. Recently, we gave in [5, Theorem 2] an alternative proof of $[10$, Assertion $(*)]$ using a reflection argument. This paper gives another instance of two different proofs.

\section{Kummer Duality}

Let $\Delta$ be a finite abelian group whose order is odd. Let $\varphi$ be a $\overline{\mathbf{Q}}_{2}$-valued character of $\Delta$ of order $d=d_{\varphi}$. Denote by $e_{\varphi}$ the idempotent of the group ring $\mathbf{Z}_{2}[\Delta]$ corresponding to $\varphi$ :

$$
e_{\varphi}=\frac{1}{|\Delta|} \sum_{\delta \in \Delta} \operatorname{Tr}\left(\varphi\left(\delta^{-1}\right)\right) \delta .
$$

Here, $\operatorname{Tr}$ is the trace map from $\mathbf{Q}_{2}\left(\zeta_{d}\right)$ to $\mathbf{Q}_{2}, \zeta_{d}$ being a primitive $d$ th root of unity. For a module $X$ over $\mathbf{Z}_{2}[\Delta]$, we denote by $X(\varphi)$ the $\varphi$-component $X^{e_{\varphi}}$ (or $e_{\varphi} X$ ). Let $\mathcal{O}_{\varphi}=\mathbf{Z}_{2}[\varphi]$ be the subring of $\overline{\mathbf{Q}}_{2}$ generated by the values of $\varphi$ over $\mathbf{Z}_{2}$. Then the $\varphi$-component $X(\varphi)$ is naturally regarded as an $\mathcal{O}_{\varphi}$-module. We choose a complete set $\Gamma=\Gamma_{\Delta}$ of representatives of the $\mathbf{Q}_{2}$-conjugacy classes of the $\overline{\mathbf{Q}}_{2}$-valued characters of $\Delta$. Then we have a canonical decomposition

$$
X=\bigoplus_{\varphi} X(\varphi)
$$

where $\varphi$ runs over the characters in $\Gamma$.

Let $T / N$ be an abelian extension over a number field $N$ with $2 \nmid[T: N]$, and let $\Delta=\operatorname{Gal}(T / N)$. Let $L / T$ be a pro-2 abelian extension which is Galois over $N$. Let $G=\operatorname{Gal}(L / T)$. Then we can naturally regard $G$ as a module over $\mathbf{Z}_{2}[\Delta]$. For a character $\varphi \in \Gamma=\Gamma_{\Delta}$, we denote by $L(\varphi)$ the intermediate field of $L / T$ corresponding to $\bigoplus_{\psi}^{\prime} G(\psi)$ by Galois theory where $\psi$ runs over the characters in $\Gamma$ with $\psi \neq \varphi$. Then we have a natural isomorphism $\operatorname{Gal}(L(\varphi) / T) \cong G(\varphi)$ of $\mathbf{Z}_{2}[\Delta]$-modules.

Now, assume that the extension $L / T$ is of exponent 2. Let $V$ be the subgroup of $T^{\times} /\left(T^{\times}\right)^{2}$ such that $L=T\left(v^{1 / 2} \mid[v] \in V\right)$. Here, for a multiplicative abelian group $X$ and an element $x \in X,[x]$ denotes the class in $X / X^{2}$ containing $x$. We can naturally regard $V$ as a module over $\mathbf{Z}_{2}[\Delta]$. The Kummer pairing

$$
V \times G \rightarrow \mu_{2}=\{ \pm 1\} ; \quad([v], g) \mapsto\langle v, g\rangle=\left(v^{1 / 2}\right)^{g-1}
$$


is nondegenerate and satisfies a relation $\left\langle v^{\delta}, g^{\delta}\right\rangle=\langle v, g\rangle$ for $[v] \in V, g \in G$ and $\delta \in \Delta$. Because of this relation, the pairing induces a nondegenerate subpairing

$$
V\left(\varphi^{-1}\right) \times G(\varphi) \rightarrow \mu_{2} .
$$

Thus, we obtain the following lemma, which we repeatedly use in this paper.

Lemma 1. Under the above setting, the Galois group $\operatorname{Gal}(L(\varphi) / T)$ is canonically isomorphic to $G(\varphi)$ as $\mathbf{Z}_{2}[\Delta]$-modules, and

$$
L(\varphi)=T\left(v^{1 / 2} \mid[v] \in V\left(\varphi^{-1}\right)\right) .
$$

\section{Lemmas}

We use the same notation as in Theorem 2. In particular, $\varphi$ is a nontrivial $\overline{\mathbf{Q}}_{2}$-valued character of $\Delta=\operatorname{Gal}(F / \mathbf{Q})=\operatorname{Gal}(K / k)$. For a number field $N, \mathcal{O}_{N}$ denotes the ring of integers of $N$. Let $E=E_{K^{+}}=\mathcal{O}_{K^{+}}^{\times}$be the group of units of $K^{+}$. We put $P^{+}=\operatorname{Gal}\left(k^{+} / \mathbf{Q}\right)$ so that $\operatorname{Gal}\left(K^{+} / \mathbf{Q}\right)=P^{+} \times \Delta$. We put

$$
\mathfrak{X}=\left(K^{+}\right)^{\times} /\left(\left(K^{+}\right)^{\times}\right)^{2}
$$

for brevity.

Lemma 2. Under the above setting, if $A_{\infty}^{+}(\varphi)$ is trivial, then both of $A^{+}(\varphi)$ and $A^{+}\left(\varphi^{-1}\right)$ are trivial.

Proof. Let $K_{>0}^{+}$be the subgroup of $\left(K^{+}\right)^{\times}$consisting of totally positive elements. Let $E_{+}=E \cap K_{>0}^{+}$, and $E_{0}$ be the subgroup of $E$ consisting of units $\varepsilon$ satisfying the congruence $\varepsilon \equiv u^{2} \bmod 4$ for some $u \in K^{+}$. We have a natural exact sequence

$$
\{0\} \rightarrow\left(K^{+}\right)^{\times} / E K_{>0}^{+} \rightarrow A_{\infty}^{+} \rightarrow A^{+} \rightarrow\{0\}
$$

compatible with the action of $\Delta$. We see that $\left(\left(K^{+}\right)^{\times} / E K_{>0}^{+}\right)(\varphi)$ is trivial if and only if $\left(E_{+} / E^{2}\right)(\varphi)$ is trivial. This is because (i) the Galois module $\left(K^{+}\right)^{\times} / K_{>0}^{+}$ is isomorphic to $\mathbf{F}_{2}\left[P^{+} \times \Delta\right]$ via the sign map, and (ii) the $\mathcal{O}_{F}$-module $\left(E / E^{2}\right)(\varphi)$ is isomorphic to $\left(\mathcal{O}_{\varphi} / 2 \mathcal{O}_{\varphi}\right)^{\oplus r}$ with $r=\left|P^{+}\right|$by a theorem of Minkowski on units of a Galois extension (cf. Narkiewicz [8, Theorem 3.26a]). Here, $\mathbf{F}_{2}$ is a finite field of 2 elements. Since $A_{\infty}^{+}(\varphi)$ is trivial, it follows from (1) that $A^{+}(\varphi)=\{0\}$ and $\left(\left(K^{+}\right)^{\times} / E K_{>0}^{+}\right)(\varphi)=\{0\}$. From the latter, it follows that

$$
\left(E_{+} / E^{2}\right)(\varphi)=\{0\} .
$$


Let $H$ be the class field of $K^{+}$corresponding to the class group $A^{+} / A^{+2}$, and $V$ the subgroup of $\mathfrak{X}$ such that $H=K^{+}\left(v^{1 / 2} \mid[v] \in V\right)$. We see that

$$
\begin{aligned}
\left(E\left(\left(K^{+}\right)^{\times}\right)^{2} /\left(\left(K^{+}\right)^{\times}\right)^{2}\right) \cap V & =\left(E_{+} \cap E_{0}\right)\left(\left(K^{+}\right)^{\times}\right)^{2} /\left(\left(K^{+}\right)^{\times}\right)^{2} \\
& =\left(E_{+} \cap E_{0}\right) / E^{2}
\end{aligned}
$$

by using Exercise 9.3 of Washington [11]. For each $[v]$ in $V$, we have $v \mathcal{O}_{K^{+}}=\mathfrak{A}^{2}$

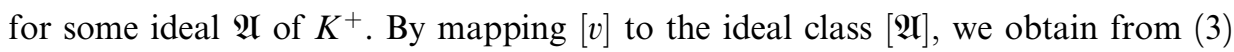
the following Kummer sequence

$$
\{0\} \rightarrow\left(E_{+} \cap E_{0}\right) / E^{2} \rightarrow V \rightarrow A^{+},
$$

which is compatible with the action of $\Delta$. Assume that $A^{+}\left(\varphi^{-1}\right)$ is nontrivial. Then it follows from Lemma 1 that $V(\varphi)$ is nontrivial. However, as $A^{+}(\varphi)$ is trivial, we see from the above Kummer sequence that $\left(\left(E_{+} \cap E_{0}\right) / E^{2}\right)(\varphi)$ is nontrivial, and hence $\left(E_{+} / E^{2}\right)(\varphi)$ is nontrivial. This contradicts (2).

In [4, Lemma 2], we showed the following assertion by effectively using the nontriviality of $\varphi$.

Lemma 3. Under the above setting, the natural map $A^{+}(\varphi) \rightarrow A(\varphi)$ is injective.

We define subgroups $\bar{A}^{+}$and $\bar{A}$ of $A^{+}$and $A$, respectively, by

$$
\bar{A}^{+}=\bigoplus_{\varphi}^{\prime} A^{+}(\varphi) \quad \text { and } \quad \bar{A}=\bigoplus_{\varphi}^{\prime} A(\varphi)
$$

where $\varphi$ runs over the nontrivial characters in $\Gamma=\Gamma_{\Delta}$. By Lemma 3, we can regard $\bar{A}^{+}$as a subgroup of $\bar{A}$. Then we put

$$
A^{*}=\bar{A} / \bar{A}^{+},
$$

which we naturally regard as a $\mathbf{Z}_{2}[\Delta]$-module. Let $\varphi_{0}$ be the trivial character of $\Delta$. Though the structures of the two minus class groups $A^{-} / A^{-}\left(\varphi_{0}\right)$ and $A^{*}$ are slightly different in general, we can easily show that $\left|A^{-}(\varphi)\right|=\left|A^{*}(\varphi)\right|$. In particular, $A^{-}(\varphi)$ is trivial if and only if $A^{*}(\varphi)$ is trivial. Let $M / K, M^{-} / K$ and $M_{\infty}^{+} / K^{+}$be the class fields corresponding to the class groups $A, A^{*}$ and $A_{\infty}^{+}$, respectively. Regarding the Galois groups $\operatorname{Gal}(M / K), \operatorname{Gal}\left(M^{-} / K\right)$ and 
$\operatorname{Gal}\left(M_{\infty}^{+} / K^{+}\right)$as modules over $\Delta=\operatorname{Gal}(K / k)=\operatorname{Gal}\left(K^{+} / k^{+}\right)$, we define the intermediate fields $M(\varphi), M^{-}(\varphi)$ and $M_{\infty}^{+}(\varphi)$ as in Section 2 for each nontrivial character $\varphi \in \Gamma$. In other words, $M(\varphi) / K, M^{-}(\varphi) / K$ and $M_{\infty}^{+}(\varphi) / K^{+}$are the class fields corresponding to $A(\varphi), A^{*}(\varphi)$ and $A_{\infty}^{+}(\varphi)$, respectively.

\section{Lemma 4. If $A^{-}(\varphi)$ is trivial, then $A_{\infty}^{+}(\varphi)$ is trivial.}

Proof. First, we show that $A^{+}(\varphi)=\{0\}$ using an argument in [7]. Because of Lemma 3 and the definition of $A^{-}$, we see that $A^{-}(\varphi)$ is the subgroup of $A(\varphi)$ consisting of classes $c$ with $c^{J}=c^{-1}$. Here, $J$ denotes the complex conjugation. Let $B^{+}$be the elements $c$ of $A^{+}(\varphi)(\subseteq A(\varphi))$ with $c^{2}=1$. For each $c \in B^{+}$, we have $c^{J}=c=c^{-1}$. It follows that $B^{+} \subseteq A^{-}(\varphi)$. As $A^{-}(\varphi)=\{0\}$, this implies that $A^{+}(\varphi)=\{0\}$. It follows that $A(\varphi)=\{0\}$ and hence $M(\varphi)=K$. Now assume that $A_{\infty}^{+}(\varphi)$ is nontrivial. Then there exists a quadratic subextension $N_{0} / K^{+}$ of $M_{\infty}^{+}(\varphi) / K^{+}$. We see that $N_{0} \cap K=K^{+}$because $\varphi$ is nontrivial. Therefore, it follows that $N_{0} K / K$ is an unramified quadratic extension contained in $M(\varphi)$, which is a contradiction.

\section{Proof of Theorem 2}

We use the same notation as in the previous sections. Replacing $F$ with the abelian field corresponding to $\operatorname{ker} \varphi$, we may as well assume that the Galois group $\Delta=\operatorname{Gal}(F / \mathbf{Q})$ is cyclic, and $\varphi: \Delta \rightarrow \overline{\mathbf{Q}}_{2}^{\times}$is injective. Further, we put

$$
\psi=\varphi^{-1}
$$

for simplicity. Let $h_{0}$ (resp. $h_{1}$ ) be the 2-part (resp. odd part) of the class number $h_{K}$ of $K$, and $h_{0}^{\prime}$ the least common multiple of $h_{0}$ and 2. We choose an element $\tilde{e}_{\varphi} \in \mathbf{Z}[\Delta]$ so that $\tilde{e}_{\varphi} \equiv e_{\varphi} \bmod h_{0}^{\prime}$ and the coefficients of $\tilde{e}_{\varphi}$ are multiple of $h_{1}$. We choose $\tilde{e}_{\psi} \in \mathbf{Z}[\Delta]$ in a similar way. We fix an element $d \in\left(k^{+}\right)^{\times}$such that

$$
k=k^{+}\left(d^{1 / 2}\right)
$$

Proof OF THE "ONLY IF"-PART. Assume that $A^{-}(\varphi)=\{0\}$. By Lemma 4, we already know that $A_{\infty}^{+}(\varphi)=\{0\}$. Hence, it suffices to show that $\varphi(p) \neq 1$ for any prime number $p$ in $S$. Assume that $\varphi(p)=1$ for some $p \in S$. Let $\wp$ be a prime ideal of $k^{+}$over $p$. As $\varphi$ is injective, the assumption $\varphi(p)=1$ implies that $\wp$ splits completely in $K^{+}$. We choose a prime ideal $\mathfrak{P}$ of $K^{+}$over $\wp$. As $A_{\infty}^{+}(\varphi)=\{0\}$, it follows from Lemma 2 that $A^{+}(\varphi)=\{0\}$. Hence, we have $\mathfrak{P}^{\tilde{e}_{\varphi}}=\pi \mathcal{O}_{K^{+}}$for some 
element $\pi$ in $K^{+}$. The ideal $\mathfrak{P}^{\tilde{e}_{\varphi}^{2}}$ is not a square of an ideal of $K^{+}$because $\tilde{e}_{\varphi}^{2} \equiv e_{\varphi} \not \equiv 0 \bmod 2$ and $\wp$ splits completely in $K^{+}$. In particular, $\pi^{\tilde{e}_{\varphi}}$ is not a square in $\left(K^{+}\right)^{\times}$. Let

$$
N_{\psi}=K^{+}\left(\left(\pi^{\tilde{e}_{\varphi}}\right)^{1 / 2}\right) .
$$

From the above, $N_{\psi} / K^{+}$is a quadratic extension. As $\varphi$ is nontrivial, we see that $N_{\psi} \cap K=K^{+}$.

Let $\tilde{\mathfrak{P}}$ be the prime ideal of $K$ over $\mathfrak{P}$. As $p \in S$, we have $\mathfrak{P}=\tilde{\mathfrak{P}}^{2}$. We have $A(\varphi)=\{0\}$ since $A^{-}(\varphi)$ and $A^{+}(\varphi)$ are both trivial. Hence, $\tilde{\mathfrak{P}}^{\tilde{e}_{\varphi}}=x \mathcal{O}_{K}$ for some $x \in K^{\times}$. It follows that $\pi=\varepsilon x^{2}$ for some unit $\varepsilon$ of $K$. Thus, we see that

$$
N_{\psi} K=K\left(\left(\pi^{\tilde{e}_{\varphi}}\right)^{1 / 2}\right)=K\left(\left(\varepsilon^{\tilde{e}_{\varphi}}\right)^{1 / 2}\right)
$$

and that this is a $(2,2)$-extension over $K^{+}$. Let $J \in \mathrm{Gal}\left(K / K^{+}\right)$be the complex conjugation. Then, as $N_{\psi} K / K^{+}$is a Galois extension, we see that $\left(\varepsilon^{\tilde{e}_{\varphi}}\right)^{J}=\varepsilon^{\tilde{e}_{\varphi}} \eta^{2}$ for some unit $\eta$ of $K$. Hence, we can extend the automorphism $J$ to that of $N_{\psi} K$ by

$$
\tilde{J}:\left(\varepsilon^{\tilde{e}_{\varphi}}\right)^{1 / 2} \mapsto\left(\varepsilon^{\tilde{e}_{\varphi}}\right)^{1 / 2} \eta
$$

Since $N_{\psi} K / K^{+}$is a $(2,2)$-extension, $\tilde{J}^{2}$ is the trivial automorphism and hence we obtain $\eta \eta^{J}=1$. It follows that $\left(\varepsilon^{\tilde{e}_{\varphi}} \eta\right)^{J}=\varepsilon^{\tilde{e}_{\varphi}} \eta$, and hence $\xi=\varepsilon^{\tilde{e}_{\varphi}} \eta \in\left(K^{+}\right)^{\times}$. We see that $\eta$ is a root of unity in $K$ by the relation $\eta \eta^{J}=1$ and a theorem ([11, Theorem 4.12]) on units of a CM field. Hence, $K\left(\left(\eta^{\tilde{e}_{\varphi}}\right)^{1 / 2}\right) / \mathbf{Q}$ is an abelian extension. However, since $\varphi$ is nontrivial, we observe using Lemma 1 that this extension would be non-abelian if the class $\left[\eta^{\tilde{e}_{\varphi}}\right]$ in $K^{\times} /\left(K^{\times}\right)^{2}$ were nontrivial. Therefore, it follows that the class $\left[\eta^{\tilde{e}_{\varphi}}\right]$ is trivial and hence

$$
N_{\psi} K=K\left(\left(\varepsilon^{\tilde{e}_{\varphi}^{2}}\right)^{1 / 2}\right)=K\left(\left(\xi^{\tilde{e}_{\varphi}}\right)^{1 / 2}\right) .
$$

Then we obtain $\pi^{\tilde{e}_{\varphi}}=\xi^{\tilde{e}_{\varphi}} y^{2}$ or $(d \xi)^{\tilde{e}_{\varphi}} y^{2}$ for some $y \in\left(K^{+}\right)^{\times}$. As $\varphi$ is nontrivial and $d \in\left(k^{+}\right)^{\times}$, we see that $d^{\tilde{e}_{\varphi}}$ is a square in $\left(k^{+}\right)^{\times}$. Hence, $\mathfrak{P}^{\tilde{e}_{\varphi}^{2}}=\pi^{\tilde{e}_{\varphi}} \mathcal{O}_{K^{+}}$is a square of a principal ideal of $K^{+}$. This is a contradiction.

To prove the "if"-part, we need to show two more lemmas.

Lemma 5. Let $\Delta_{0}$ be a nontrivial subgroup of $\Delta$, and let $\kappa=\kappa_{\Delta_{/} \Delta_{0}}$ be the restriction map $\mathbf{Z}_{2}[\Delta] \rightarrow \mathbf{Z}_{2}\left[\Delta / \Delta_{0}\right]$. Then we have $\kappa\left(e_{\varphi}\right)=0$.

Proof. We have $\sum_{\delta \in \Delta_{0}} \varphi(\delta)=0$ since $\Delta_{0}$ is nontrivial and $\varphi$ is injective. The assertion follows from this. 
Lemma 6. Let $\mathfrak{B}$ be a prime ideal of $K^{+}$, and $\wp=\mathfrak{B} \cap k^{+}$and $p=\wp \cap \mathbf{Q}$. If $\varphi(p) \neq 1$ and $A^{+}(\varphi)=\{0\}$, then $\mathfrak{P}^{\tilde{e}_{\varphi}^{2}}=x^{2} \mathcal{O}_{K^{+}}$for some $x \in\left(K^{+}\right)^{\times}$.

Proof. Let $F_{0}$ be the decomposition field of $p$ at $F / \mathbf{Q}$, and $\Delta_{0}=$ $\operatorname{Gal}\left(F / F_{0}\right)$. As $\varphi(p) \neq 1, \Delta_{0}$ is a nontrivial subgroup of $\Delta$. We put $K_{0}^{+}=F_{0} k^{+}$ and $\mathfrak{P}_{0}=\mathfrak{B} \cap K_{0}^{+}$. Since $\mathfrak{P}_{0}$ remains prime in $K^{+}$, we see that $\mathfrak{P}^{\tilde{e}_{\varphi}}=\mathfrak{P}_{0}^{k\left(\tilde{e}_{\varphi}\right)} \mathcal{O}_{K^{+}}$ where $\kappa=\kappa_{\Delta / \Delta_{0}}$ is the restriction map in Lemma 5. By Lemma 5, we have $\kappa\left(\tilde{\boldsymbol{e}}_{\varphi}\right) \equiv 0 \bmod 2$, and hence $\mathfrak{P}^{\tilde{e}_{\varphi}}=\mathfrak{A}^{2}$ for some ideal $\mathfrak{A}$ of $K^{+}$. It follows that $\mathfrak{P}^{\tilde{e}_{\varphi}^{2}}=\left(\mathfrak{Y}^{\tilde{e}_{\varphi}}\right)^{2}$. As $A^{+}(\varphi)=\{0\}, \mathfrak{U}^{\tilde{e}_{\varphi}}$ is principal, and hence the assertion follows.

Proof OF THE "IF"-PART. Assume that (i) $A_{\infty}^{+}(\varphi)=\{0\}$ and that (ii) $\varphi(p) \neq 1$ for any prime number $p \in S$. Then we have

$$
A^{+}(\psi)=\{0\}
$$

by Lemma 2. To show the assertion, assume to the contrary that $A^{-}(\varphi)$ is nontrivial. Then the extension $M^{-}(\varphi) / K$ is nontrivial. Let $v$ be an arbitrary infinite prime divisor of $K^{+}$. By using an argument in Iwasawa [6] (or in pp. 186-187 of [11]), we see that there exists a quadratic extension $N_{0}=$ $K^{+}\left(w^{1 / 2}\right) / K^{+}$with $w \in K^{+}$which is unramified at $v$ and satisfies $N_{0} K \subseteq M^{-}(\varphi)$. In particular, $N_{0} \cap K=K^{+}$. Let $v=w^{\tilde{e}_{\psi}}$ and $N=K^{+}\left(v^{1 / 2}\right)$. Then we see that $N K=N_{0} K \subseteq M^{-}(\varphi)$ from Lemma 1 and that $N K / K^{+}$is a $(2,2)$-extension. Since the extension $N / K^{+}$is unramified outside $S$ and $\infty$, we can write

$$
v \mathcal{O}_{K^{+}}=\prod_{\mathfrak{P}} \mathfrak{P}^{a_{\mathfrak{F}}} \mathfrak{Q}^{2}
$$

for some ideal $\mathfrak{A}$ of $K^{+}$. Here, $\mathfrak{P}$ runs over the prime ideals of $K^{+}$with $\mathfrak{P} \cap \mathbf{Q} \in S$, and $a_{\mathfrak{P}}=0$ or 1 . As $[v] \in \mathfrak{X}(\psi)$, we may as well replace the Kummer generator $v$ with $v^{\tilde{e}_{\psi}^{2}}$. Then, since $A^{+}(\psi)=\{0\}$ and $\psi(p) \neq 1$ for any $p \in S$, it follows from Lemma 6 that $v \mathcal{O}_{K^{+}}=x^{2} \mathcal{O}_{K^{+}}$for some $x \in\left(K^{+}\right)^{\times}$. Therefore, we have

$$
N=K^{+}\left(\varepsilon^{1 / 2}\right)=K^{+}\left(\left(\varepsilon^{\tilde{e}_{\psi}}\right)^{1 / 2}\right)
$$

for some unit $\varepsilon$ of $K^{+}$with $[\varepsilon] \in\left(E / E^{2}\right)(\psi)$. It follows that $N / K^{+}$is unramified outside $\infty$ and $S \cap\{2\}$. Therefore, when $2 \notin S$ or $N / K^{+}$is unramified at 2 , $N / K^{+}$is unramified outside $\infty$, and hence $N \subseteq M_{\infty}^{+}(\varphi)$. Thus, we see that $A_{\infty}^{+}(\varphi)$ is nontrivial, which is a contradiction. 
Assume that $2 \in S$ and that there is a prime ideal $\mathfrak{P}$ of $K^{+}$over 2 which ramifies in $N$. Since $k=k^{+}\left(d^{1 / 2}\right)$, the quadratic subfields of the $(2,2)$-extension $N K / K^{+}$are $K, N$ and

$$
N^{\prime}=K^{+}\left((d \varepsilon)^{1 / 2}\right)=K^{+}\left(\left(d \varepsilon^{\tilde{e}_{\psi}}\right)^{1 / 2}\right) .
$$

Since $N K / K$ is unramified at $\mathfrak{P}$, and $K / K^{+}$and $N / K^{+}$are ramified at $\mathfrak{P}$, the third extension $N^{\prime} / K^{+}$is unramified at $\mathfrak{P}$. Therefore, $\operatorname{ord}_{\mathfrak{P}}(d)$ is even. This means that $\operatorname{ord}_{\wp}(d)$ is even as $\left[K^{+}: k^{+}\right]$is odd, where $\wp=\mathfrak{P} \cap k^{+}$. Then, replacing $d$ with $d y^{2}$ for some $y \in\left(k^{+}\right)^{\times}$, we may as well assume that $(d, \mathfrak{P})=1$. Since $N^{\prime} / K^{+}$is unramified at $\mathfrak{P}$, we have $d \varepsilon^{\tilde{\varepsilon}_{\psi}} \equiv u^{2} \bmod \mathfrak{P}^{2 e}$ for some $u \in K^{+}$by Exercise 9.3 of [11]. Here, $e$ is the ramification index of $\mathfrak{P}$ over $\mathbf{Q}$. Let $\Delta_{0}$ be the decomposition group of the prime 2 at $F / \mathbf{Q}$. Let $\psi_{0}=\psi_{\mid \Delta_{0}}$ and define $e_{\psi_{0}}$ and $\tilde{e}_{\psi_{0}}$ similarly to $e_{\psi}$ and $\tilde{e}_{\psi}$. Since $\mathfrak{P}$ is stable under the action of $\Delta_{0}$, we have

$$
\left(d \varepsilon^{\tilde{e}_{\psi}}\right)^{\tilde{e}_{\psi_{0}}} \equiv v^{2} \bmod \mathfrak{P}^{2 e}
$$

for some $v \in\left(K^{+}\right)^{\times}$. As $\psi(2) \neq 1, \Delta_{0}$ is nontrivial and hence $d^{\tilde{e}_{\psi_{0}}}$ is a square in $k^{+}$. Further, we have $\tilde{e}_{\psi} \tilde{e}_{\psi_{0}} \equiv \tilde{e}_{\psi} \bmod 2$ as $e_{\psi} e_{\psi_{0}}=e_{\psi}$. Therefore, we see that $\varepsilon^{\tilde{e}_{\psi}} \equiv w^{2} \bmod \mathfrak{P}^{2 e}$ for some $w \in\left(K^{+}\right)^{\times}$. This implies that $N / K^{+}$is unramified at $\mathfrak{P}$, a contradiction.

\section{Cyclotomic $\mathrm{Z}_{2}$-extension}

Let $F, \Delta$ and $\varphi$ be as in the previous sections. For an integer $n \geq 0$, let $k_{n}=\mathbf{Q}\left(\zeta_{2^{n+2}}\right), K_{n}=F k_{n}, F_{n}=K_{n}^{+}$and $\mathbf{B}_{n}=k_{n}^{+}$. As in the previous sections, we identify $\Delta$ with $\operatorname{Gal}\left(K_{n} / k_{n}\right)$ and $\operatorname{Gal}\left(F_{n} / \mathbf{B}_{n}\right)$. We write $\varphi \sim \varphi^{-1}$ when $\varphi$ and $\varphi^{-1}$ are conjugate over $\mathbf{Q}_{2}$. It is well known that the class groups $A_{\mathbf{B}_{n}, \infty}$ and $A_{k_{n}}^{-}$are trivial for all $n \geq 0$. We generalize this fact as follows.

THEOREM 3. Under the above setting, assume that

$$
\text { (C1) } \varphi \sim \varphi^{-1}, \quad(\mathrm{C} 2) \varphi(2) \neq 1, \quad(\mathrm{C} 3) A_{F}(\varphi)=\{0\} .
$$

Then the class groups $A_{F_{n}, \infty}(\varphi)$ and $A_{K_{n}}^{-}(\varphi)$ are trivial for all $n \geq 0$.

Proof. We write $A_{n, \infty}=A_{F_{n}, \infty}$ for brevity. By virtue of Theorem 2 (and the assumption (C2)), the triviality of $A_{K_{n}}^{-}(\varphi)$ follows from that of $A_{n, \infty}(\varphi)$. The assumption (C1) implies that

$$
X(\varphi)=X\left(\varphi^{-1}\right)
$$


for a $\mathbf{Z}_{2}[\Delta]$-module $X$. Further, it follows from the assumptions $(\mathrm{C} 1)$ and $(\mathrm{C} 3)$ that $A_{0, \infty}(\varphi)=\{0\}$ by [9, Théorème 2]. To show Theorem 3, assume to the contrary that $A_{n, \infty}(\varphi) \neq\{0\}$ for some $n \geq 1$. Let $M_{n, \infty}(\varphi) / F_{n}$ be the class field corresponding to $\left(A_{n, \infty} / A_{n, \infty}^{2}\right)(\varphi)$. The cyclic extension $F_{n} / F$ is of degree $2^{n}$ and unramified outside 2. Hence, using an argument in [6], we see that there exists a quadratic extension $N^{\prime}=F\left(w^{1 / 2}\right) / F$ unramified at some prime ideal of $F$ over 2 with $N^{\prime} F_{n} \subseteq M_{n, \infty}(\varphi)$. In particular, we have $N^{\prime} \cap F_{n}=F$. Put $v=w^{\tilde{e}_{\varphi}}$, and $N=F\left(v^{1 / 2}\right)$. Then, we see from Lemma 1 and (4) that $N F_{n}=N^{\prime} F_{n} \subseteq M_{n, \infty}(\varphi)$ and $N \cap F_{n}=F$. Clearly, $N / F$ is unramified outside 200 . Using Lemma 6 and (C2), (C3), we can show that $N=F\left(\varepsilon^{1 / 2}\right)$ for some unit $\varepsilon$ of $F$ with $[\varepsilon] \in$ $\left(E / E^{2}\right)(\varphi)$ by an argument similar to that in the proof of the "if part" of Theorem 2. Here, $E=\mathcal{O}_{F}^{\times}$.

We already know that the narrow class group $A_{0, \infty}(\varphi)$ is trivial. Hence, the quadratic extension $N / F$ is ramified at some prime ideal $\mathfrak{P}$ of $F$ over 2 . We define an integer $\pi_{j}$ of $F_{j}$ inductively by $\pi_{0}=2$ and $\pi_{j}=2+\sqrt{\pi_{j-1}}$ for $j \geq 1$. Then $\pi_{j}$ is a local parameter of each prime ideal of $F_{j}$ over 2 and $F_{j+1}=F_{j}\left(\pi_{j}^{1 / 2}\right)$. Since $N F_{n} / F_{n}$ is unramified at 2 , there exists some $j$ with $0 \leq j \leq n-1$ such that $N F_{j} / F_{j}$ is ramified and $N F_{j+1} / F_{j+1}$ is unramified at the primes ideals over $\mathfrak{P}$. This implies that the intermediate extension $F_{j}\left(\left(\varepsilon \pi_{j}\right)^{1 / 2}\right) / F_{j}$ of the $(2,2)$-extension $N F_{j+1} / F_{j}$ is unramified at the primes ideals over $\mathfrak{P}$. However, this is impossible because $\varepsilon$ is a unit and $\pi_{j}$ is a local parameter of the prime ideals of $F_{j}$ over 2 .

\section{References}

[ 1 ] Armitage, J. V. and Fröhlich, A., Classnumbers and unit signature, Mathematika 14 (1967), 94-98.

[2] Conner, P. E. and Hurrelbrink, J., Class Number Parity, World Scientific, Singapore, 1988.

[ 3 ] Hasse, H., Über die Klassenzahl abelscher Zahlkörper (Reprint of the first edition), Springer, Berlin, 1985.

[4] Ichimura, H., Class number parity of a quadratic twist of a cyclotomic field of prime power conductor, Osaka J. Math. 50 (2013), 563-572.

[5] Ichimura, H., On a duality of Gras between totally positive and primary cyclotomic units, to appear in Math. J. Okayama Univ.

[6] Iwasawa, K., A note on class numbers of algebraic number fields, Abh. Math. Sem. Univ. Hamburg 20 (1956), 257-258.

[ 7 ] Iwasawa, K., A note on ideal class groups, Nagoya Math. J. 27 (1966), 239-247.

[8] Narkiewicz, W., Elementary and Analytic Theory of Algebraic Numbers (3rd. ed.), Springer, Berlin, 2004.

[9] Oriat, B., Relation entre les 2-groupe des classes d'idéaux au sens ordinaire et restreint de certains corps nombres, Bull. Soc. Math. France 104 (1976), 301-307.

[10] Taylor, M. J., Galois module structure of classgroups and units, Mathematika 22 (1975), $156-160$. 
[11] Washington, L. C., Introduction to Cyclotomic Fields (2nd. ed.), Springer, New York, 1997.

Faculty of Science, Ibaraki University Bunkyo 2-1-1, Mito, 310-8512, Japan

E-mail: hichimur@mx.ibaraki.ac.jp 